\section{P4.021 ASSESSMENT OF STIGMA AMONG PATIENTS OF HEPATITIS B AND C}

doi:10.1136/sextrans-2013-051184.0919

II Rafique, 'M A N Saqib, ${ }^{2}$ S Siddiqui, 'M A Munir, ${ }^{3} \mathrm{~N}$ Javed, ${ }^{3} \mathrm{~S}$ Naz, ${ }^{4} \mathrm{Z}$ Z Tirmizi, ${ }^{1} \mathrm{H}$ Qureshi. 'Pakistan Medical Research Council, Islamabad, Pakistan; ${ }^{2}$ Pakistan Institute of Medical Sciences, Islamabad, Pakistan; ${ }^{3}$ Central Research Center, PMRC, NIHI, Islamabad, Pakistan; ${ }^{4}$ Federal Goverenment Services Hospital, Islamabad, Pakistan

Background Stigma has negative effects on self-esteem resulting in decreased quality of life in affected individuals and is a major obstruction in the testing, management and treatment. This study was conducted to assess stigma among hepatitis B and C patients in our society.

Methods A total of 140 indoor and outpatient hepatitis B/C positive patients were enrolled from tertiary care hospitals of Islamabad and Rawalpindi, Pakistan. Semi-structured questionnaire was used for interview and focus group discussions (FGD's) with patients and their relatives were also conducted.

Results Majority of patients (81\%) were HCV. When patients were asked how they got hepatitis, about $58 \%$ had no idea. Similarly $59 \%$ patients got information about hepatitis after getting diagnosed. Majority (83\%) was worried and in 74\%, life style was affected due to weakness and emotional disturbances. Martial relationship was affected in about half of the cases.

About $66 \%$ patients had fear of disease transmission to others and among them $64 \%$ believed that this can be transmitted by sharing eating utensils. When participants were asked about sharing of utensils and towels, eating, sitting and shaking hand, it was found that family members, relatives and friend were hesitant and in some cases, patients were ignored.

During focus group discussions, it was found that in Pakistan, hepatitis $B$ and $C$ patients had variety of stigmas such as fear of transmission of disease, social isolation, and discrimination in getting job, break up of engagement, divorce. Similarly patients were having different conceptions about disease treatment as one quarter was taking medication other than allopathic.

Conclusion This study showed the presences of misconception and stigma in society which need to be addressed by public awareness programmes.

\section{P4.022 A SEXUAL AFFILIATION NETWORK OF SWINGERS AND THE SPREAD OF STI, A TWO-MODE NETWORK APPROACH}

doi:10.1136/sextrans-2013-051184.0920

\begin{abstract}
1,2A M Niekamp, ${ }^{1,2}$ C J P A Hoebe, ' ${ }^{2} \mathrm{~L}$ A G Mercken, 1,2N H T M Dukers-Muijrers. ' $P$ Public Health Service South Limburg, Geleen, The Netherlands; ${ }^{2}$ School of Public Health and Primary Care (CAPHRI), Maastricht University, Maastricht, The Netherlands
\end{abstract}

Background An increasing body of evidence indicates venues where people recruit sex partners have a role in spread of STIs. Swingers recruit their sex partners by physical venues (clubs) and virtual venues (websites), forming so-called sexual affiliation networks. Objective of the present paper is to examine how these sexual affiliation networks of swingers can be relevant for STI prevention.

Methods Participants of our swinger's cohort were followed using questionnaires. We used both conventional epidemiological and social network methods (descriptive and Exponential Random Graph Models (ERGM)) for the analysis. Because couples swing together, they were found equal in their choices of venues and taken as a swing unit (SU).

Results The 57 SU frequented 13 (33.3\%) clubs and 26 (66.7\%) websites; $59.6 \%(n=34)$ of the SU frequented both websites and clubs, $36.8 \%(n=21)$ frequented only websites and $2(3.5 \%)$ solely clubs.
The network formed only one component (fragmentation $=0$ ): all SU and venues in the network were interconnected. The sexual affiliation network has a clear core-periphery structure, with a core of highly connected SU $(n=16)$ and venues $(n=7)$.

SU characteristics were generally not statistically significantly associated with the number of websites or clubs frequented, except for the following three: swinging years, group sex, and drug use

Drug users had a significant stronger tendency to visit websites than non-drug users, and also had a strong tendency to frequent websites more than clubs.

Conclusions The analysis of sexual affiliation networks has an important added value to conventional STI epidemiology, because the last does not assess the direct relations between actors and mechanisms of clustering. Furthermore our results show that all swingers in the network were interconnected through their affiliations and therefore STI can eventually reach everyone. Interventions that focus on the prevention of drug use should be directed to visitors of websites instead of clubs.

\section{P4.023 FACTORS CONTRIBUTING TO HIV SEROCONVERSION AMONG WOMEN IN PRIMARY RELATIONSHIPS IN UGANDA - THE WOMEN'S PERSPECTIVE}

doi:10.1136/sextrans-2013-051184.0921

\section{B Mpairwe. Joint Clinical Research Centre, Kampala, Uganda}

Background This study describes the existing socio-economic and behavioural factors that contributed to the risk of HIV seroconversion among women in the HC-HIV study in Uganda.

Methods It was a descriptive cross sectional study looking at socio-economic and behavioural factors that contributed to HIV seroconversion among women. The study used both qualitative and quantitative data collection methods.

Results Almost a third of these women had separated within six months to seroconversion. Twenty nine women $(30.21 \%, \mathrm{n}=96)$ seroconverted after separating and picking on a new sexual partner within six months to seroconversion. Polygamy accounted for almost a third (31.25\%) of marriages. In total ( $42.71 \%, n=96)$ of the women reported having multiple sexual partners at the time of seroconversion. Twenty three women said that existing financial pressures influenced their decisions on how to get additional financial support and $(73.91 \%, n=23)$ of these women say they picked on a new sexual partner for financial support. Having more than one sexual partner, mean age 25 yrs (Standard deviation 5.02), having one sexual partner, mean age 28yrs (Standard deviation 5.60). $\mathrm{P}=0.0043$. A third of women with no education had more than one sexual partner, $57.78 \%$ for those with primary education, $30 \%$ for those with secondary education and $25 \%$ for those with tertially education. $\mathrm{P}=0.035$. Women not employed and were housewives were $51 \%$ less likely to have multiple sexual partners (OR 0.5101449 , CI $0.1911013-1.323363, \mathrm{P}=0.139$ ).

Conclusions There was a close relationship and inter linkage between the factors. In low income countries like Uganda, where education and economic empowerment is still low, and financial as well as cultural dependence of women on their partners is the norm, some women would rather risk seroconverion than loose their only available socio-economic security/their spouses.

\section{P4.024 DO NEW MEDIA AFFECT ADOLESCENT SEXUAL ATTITUDES AND BEHAVIOURS? A SYSTEMATIC REVIEW}

doi:10.1136/sextrans-2013-051184.0922

'L Watchirs Smith, 'R Guy, ${ }^{2} \mathrm{~L}$ Degenhardt, ${ }^{3} \mathrm{~J}$ Richters, ${ }^{4} \mathrm{~S}$ Robbins, ' $\mathrm{J}$ Kaldor, ${ }^{5} \mathrm{C}$ Lumby, ${ }^{6} \mathrm{R}$ Skinner, ${ }^{1} \mathrm{~B}$ Liu. ${ }^{1}$ Kirby Institute, UNSW, Sydney, Australia; ${ }^{2}$ National Drug and Alcohol Research Centre, UNSW, Sydney, Australia; ${ }^{3}$ School of Public Health and Community Medicine, UNSW, Sydney, Australia; ${ }^{4}$ Western Sydney Sexual Health 
Centre, Sydney University, Sydney, Australia; '5Journalism and Media Research Centre UNSW, Sydney, Australia; ' 6 Sydney University Discipline of Paediatrics and Child Health The Children's Hospital at Westmead, Sydney, Australia

Introduction There is considerable public concern that new media (including the Internet and mobile phones) could be exposing young people to high levels of sexual content and may impact risky behaviour and/or risk for sexually transmissible infections.

Methods The review was conducted in accordance with the PRISMA guidelines. Medline, EMBASE and PsychINFO were searched to the end of August 2012. Articles were included if they described the statistical association between exposure to sexual content in new media (viewing or engaging) and sexual attitudes or behaviours in young people (defined as $<25$ years).

Results There were 3834 articles identified, and five met the inclusion criteria: all were cross-sectional designs (four convenience samples), with the largest study containing 6054 participants. In four studies the exposure was viewing sexually explicit websites (SEW); a range of outcomes were assessed however each outcome variable was only measured in one or two of the included studies. Viewing SEW was significantly associated with: first intercourse $<15$ years of age, ever having a sexual partner, $>1$ partner in last 3 months, multiple lifetime partners, unprotected sex at last sex, drug and alcohol use at last sex, acceptance of casual sexual relationships, having casual sexual relationships, notions of women as sex objects, and approval of extra-marital sex. "Sexting" (sending or receiving sexual texts) was the exposure in the fifth study and was associated with ever having unprotected sex.

Conclusion The relationship between SEW and sexual behaviours and attitudes was inconsistent. Engagement with sexual media appeared to be associated with markers of higher sexual interest and/or activity, but study size, methodological approach and inconsistencies in outcome measures prevented us from drawing conclusions regarding causality. No study explored associations with positive aspects of sexual development (e.g. sexual communication, sexual assertiveness, relationship quality). Further research in this emerging area is needed.

\section{P4.025 CONCEPTUALIZATION OF ANAL SEX BEHAVIOUR WITHIN SEXUAL PARTNER RELATIONSHIPS FOR MODIFICATION OF EVIDENCE-BASED ADOLESCENT SEXUAL HEALTH PROMOTION INTERVENTIONS}

doi:10.1136/sextrans-2013-051184.0923

IJ D Champion, ${ }^{2} \mathrm{C}$ Roye. ${ }^{1}$ The University of Texas at Austin, Austin, TX, United States, 2Hunter, CUNY, New York, NY, United States

Background Understanding of the context of anal sex behaviour among Black and Hispanic adolescent women with a history of sexually transmitted infection (STI/HIV) has public health implications for health promotion and risk reduction behavioural interventions.

Methods African-American ( $\mathrm{n}=94,16.8 \%$ ) and Mexican-American ( $n=465,83.2 \%$ ) women (14-18 years) with histories of sexual risk behaviour, STI or violence, recruited from metropolitan sexual health clinics for participation in a clinical trial for behavioural interventions, completed semi-structured interviews to assess psychosocial and situational factors associated with high sexual risk behaviour, substance use, STI/HIV acquisition, and violence occurrence at study entry.

Results Anal sex varied by ethnicity (24.1\% Mexican-American, $10.6 \%$ African-American, $p=0.004)$ and was associated with more $(p<0.05)$ run-away, school dropout, sexual partners, bisexuality, sex for favours, money or friends with benefits, group sex, substance and alcohol use, sex when high, history of STI, withdrawal for birth control and psychological distress. More (94.3\% vs. $85.1 \%$ ) and higher levels of violence (8.37 vs. 6.38) overall, and by comparisons of sexual and physical violence was identified. Age at first anal sex (15.85 years) with partner age (18.43 years), ever condom use for anal sex (38\%), anal sex past year $(79.5 \%)$, past 6 months $(61.2 \%)$ past 3 months (36.7\%) with limited condom use (40.8\%) was selfreported. Fewer women reporting anal sex could stop having sex with partners having sex with others ( $64.7 \%$ vs. $76.2 \%)$ while more believed it's okay to have a man on the side for more variety/sex (43.9\% vs. $18.3 \%$ ), he fools around so why shouldn't I? (25.8\% vs. $15.1 \%)$ or I get high and lose control (24.2\% vs. $14.5 \%)$.

Conclusions Conceptualization of anal sex behaviour within partner relationships is important for modification of evidence-based sexual health promotion interventions to enhance efficacy for prevention of violence, substance use, unintended pregnancy and STI/ HIV among ethnic minority adolescent women.

\section{P4.026 INDIAN BOARDING SCHOOL EXPERIENCE AND HIV SEXUAL RISK BEHAVIORS AMONG URBAN TWO-SPIRIT AMERICAN INDIAN/ALASKA NATIVES}

doi:10.1136/sextrans-2013-051184.0924

T Evans-Campbell, C R Pearson, K L Walters. University of Washington, Seattle, WA United States

Background As part of a systemic effort of assimilation between the late $19^{\text {th }}$ and mid-20 $0^{\text {th }}$ centuries, thousands of American Indian/ Alaska Native (AIAN) children were placed in residential Indian boarding schools. To understand the impact of forced attendance in Indian boarding schools on HIV risk, we explored sexual risk behaviours, STI diagnosis, and substance misuse among AIAN two-spirit (gay, lesbian, bisexual, and transgender) adults who had attended Indian boarding school as children compared to those with no history of boarding school.

Methods The current investigation was part of a comprehensive multi-site, cross-sectional national health survey of 447 AIAN two-spirit people from seven metropolitan areas in the U.S. To minimise selection bias, multiple sampling strategies were used including targeted, partial network, and respondent-driven sampling (RDS) techniques.

Results Eighty-two (22.9\%) respondents had a history of Indian boarding school attendance. Compared to others, respondents who attended boarding school were more likely to have a diagnosis of alcohol abuse or dependence ( $58.5 \%$ vs. $44.9 \%, p<0.05)$ and reported higher illicit drug use in the past 12 months including: club drugs $(18.3 \%$ vs. $9.6 \%, p<0.05)$, crack-cocaine $(43.9 \%$ vs. $29.6 \%, p<0.01)$, and erectile dysfunction drugs $(7.3 \%$ vs. $2.7 \%, p<0.05)$. Former boarding school attendees also reported more lifetime partners (5.9 vs. $5.4, p<0.05)$, were more likely to have had an STI $(42.7 \%$ vs. $30.7 \%, p<0.05)$, and were more likely to have ever traded sex for drugs, money, or food (56.8\% vs. $33.5 \%, p<0.001)$.

Conclusion Findings from this study provide some of the first data on boarding school experience and HIV risk among an urban twospirit AIAN community sample and underscore the need for increased HIV/STI prevention efforts in AIAN communities. In addition, advanced statistical models are needed that identify mediational pathways to health outcomes and risk behaviours.

\section{P4.027 THE PRACTISE OF ANAL SEX AMONG THE THE FEMALE SEX WORKERS IN THE WESTERN PART OF ASSAM, INDIA}

doi:10.1136/sextrans-2013-051184.0925

A D Sharma. Dermacare Clinic, Bongaigaon, India

Background Unprotected anal sex is associated with the highest risk of transmission of sexually transmitted infections (STIs) and human immunodeficiency virus (HIV). This is due to the delicate nature of the rectal mucosa and anal sphincter. Any from of trauma 